\title{
Las mujeres y el manejo de un síntoma: de la valoración a la selección
}

\author{
Carmen de la Cuesta-Benjumea, R.N ., M. Sc., Ph.D..$^{(1)}$
}

\section{De la Cuesta-Benjumea C. Las mujeres y el manejo de un síntoma: de la valoración a la selección. Salud Publica Mex 1999;41:124-129.}

\section{Resumen}

Objetivo. Describir, desde el punto de vista de los pacientes, el proceso que siguen éstos para conseguir ayuda médica. Material y métodos Los hallazgos aquí reportados forman parte de un estudio cualitativo sobre la salud de las mujeres realizado en Colombia. Los datos se recogieron por medio de entrevistas semiestructuradas: la teoría fundada guió sus análisis. Resultados Ante un síntoma o signo, las mujeres primero lo valoran, luego miden sus propias capacidades y evalúan sus opciones y, por último, seleccionan a quien pueda ayudarlas. Estas tres etapas forman parte del proceso de enfrentar un síntoma o signo. Conclusiones Los profesionales de la salud han de ser sensibles a la experiencia subjetiva de los pacientes y deben ser capaces de reconocer su papel en el diagnóstico y el cuidado de la salud de los mismos.

Palabras clave: salud de las mujeres; servicios de salud/utilización; Colombia

\author{
De la Cuesta-Benjumea C. \\ How women face a medical symptom \\ from evaluation to health care selection. \\ Salud Publica Mex 1999;41:124-129.
}

\section{A bstract}

Objective. To describe, from the patients' point of view, the process they follow from the moment they face a symptom until they seek medical help. Material and methods. Findings reported here are part of a qualitative study on the health of women from Colombia. Data were collected by semistructured interviews. The analysis was guided by grounded theory. Results.W hen facing a symptom, women first evaluate it, then weigh their own capacities and assess their options, and last, they chose who might help them. These three stages form part of the process followed to seek medical help. Conclusions. Health professionals should be sensitive to patients' subjective experience and acknowledge their role in the diagnostic process and in health care.

Key words: women's health; health services/utilization; C olombia
D iversos estudios muestran que no existe una relación directa entre sentirse enfermo e ir al médico, ya que este fenómeno está mediado por un proceso social en el que interviene la percepción individual y la forma en que el paciente interpreta los síntomas. ${ }^{1}$ La antropología médica en particular, resalta el papel fundamental que las interpretaciones culturales representan en la experiencia y en la conducta ante la enfermedad. Así, en las décadas de los años setenta y ochenta se realizaron diversos estudios sobre los sistemas de creencias respecto a la salud y los significados que las personas atribuyen a los síntomas. Estos estudios indicaron que el significado está moldeado social y culturalmente, y que esto a su vez se constituye en una experiencia para el paciente. Se hizo evidente que las interpretaciones se guían por conceptos e ideas de considerable complejidad y variedad. ${ }^{2}$ Para clarificar esto se han ofrecido diversos modelos, de

(1) Profesora A sociada, Facultad N acional de Salud Pública, Universidad de Antioquia, Medellín, Colombia.

Fecha de recibido: 22 de septiembre de 1998 - Fecha de aprobado: 23 de noviembre de 1998 
los cuales se destaca el explicativo, de Kleinman, que permite examinar cómo las personas implicadas en el proceso clínico se organizan, e interpretan y tratan la enfermedad. ${ }^{3}$

Por otro lado, la sociología médica ha señalado que el proceso por el cual las personas buscan ayuda conlleva múltiples decisiones sobre el cuerpo y sus necesidades. Se ha comprobado que el autocuidado es más común que el tratamiento brindado por los profesionales, y que hay un sistema de salud oculto que proporciona asesoría, cuidados y guía en la búsqueda de atención profesional. ${ }^{4}$ Así, la experiencia de la enfermedad incluye un proceso de toma de decisiones que comprende varias etapas, en las que el contacto con la asistencia profesional es sólo una de ellas. ${ }^{5,6}$

No obstante, no se elige en el vacío, sino dentro del contexto social, económico e ideológico (o religioso) en el que se encuentran las personas. ${ }^{3}$

El propósito de este artículo es describir, desde el punto de vista de los pacientes, el trayecto que siguen éstos para solicitar la ayuda médica. Los hallazgos aquí notificados forman parte de un estudio cualitativo sobre la salud de las mujeres realizado en la región de Antioquia, Colombia, durante 1994. ${ }^{7}$

Los primeros estudios desarrollados en este campo fueron encuestas que mostraron el "fracaso" de los enfermos en obtener ayuda a tiempo. De hecho, desde los años cuarenta, muchos de estos acercamientos han mostrado que la mayoría de los síntomas de enfermedad se ignoran o no reciben atención médica, fenómeno que se ha denominado "iceberg de la enfermedad". 8,9 Desde los años sesenta ha tenido lugar una exploración sistemática de las variaciones en las formas en las que las personas perciben, evalúan y actúan en relación con su salud.

Se pueden distinguir dos enfoques en estas investigaciones: ${ }^{1}$ uno de ellos, el estructuralista, explora el complejo conjunto de factores estructurales que influyen en la conducta de convertirse en enfermo. En esta tradición se resaltan los rasgos comunes de grupos con valores culturales y experiencias sociales compartidos; cabe destacar los estudios que relacionan la clase social, el género y la etnia con el comportamiento respecto a la salud. ${ }^{10} \mathrm{El}$ otro enfoque, el interaccionista, enfatiza que las personas son participantes activos en todo el tiempo que dura su enfermedad. Obviamente, el enfoque estructuralista no ignora que las personas son actores, pero su interés principal se centra en los patrones sociales estables de la acción. En el interaccionismo se destaca que las personas toman decisiones de forma consciente, que sus acciones tienen un propósito y que se encuentran inmersas en redes com- plejas de negociaciones. Este enfoque es el que se ha utilizado en el presente estudio.

\section{Material y métodos}

Esta investigación surge por la necesidad de incorporar el punto de vista de las mujeres en los diagnósticos de salud que realizan los profesionales. Uno de sus objetivos fue comprender cómo consideran las mujeres los servicios de salud. Se eligió el enfoque cualitativo, pues, aplicado a este campo, toma en cuenta los múltiples significados que los individuos adjudican a su cuidado, sus diversas conductas y sus prácticas en salud. ${ }^{11}$

Veinticinco mujeres de la región de Antioquia participaron en el estudio. Lo hicieron de forma voluntaria e informada; se les garantizó el anonimato y el manejo confidencial de los datos. Debido a que los estudios cualitativos pretenden la identificación, la descripción y el análisis de fenómenos, el muestreo se realizó con base en los conceptos o categorías que aparecieron durante el análisis. A esto se le denomina muestreo téorico, ${ }^{12}$ y busca la descripción más completa del fenómeno en términos de su variación y rango. ${ }^{13}$ Del muestreo resultó un grupo de mujeres variado y equilibrado. La mayoría tenía de 20 a 39 años de edad, y sólo cinco mujeres superaban los 40 años. Catorce de ellas tenían hijos; asimismo, 12 eran casadas; 11, solteras; una estaba separada, y otra era viuda. Respecto a la actividad que desempeñaban, ocho realizaban trabajo calificado; cinco, muy calificado; cuatro, sin calificar; otras cuatro eran amas de casa, y cinco, estudiantes. Respecto a su nivel de educación, siete tenían estudios superiores, y sólo dos no habían cursado la primaria ni tenían estudios.

Los datos se recogieron por medio de 25 entrevistas individuales y semiestructuradas, ${ }^{14}$ con una duración de 30 a 40 minutos cada una; se grabaron y transcribieron en su totalidad. Se realizaron en Medellín y en pueblos de la misma región. Los lugares se seleccionaron por la facilidad del acceso a las participantes, criterio legítimo en este tipo de estudios. ${ }^{15}$ Para el análisis de los datos se recurrió a la teoría fundada, ${ }^{16}$ y esto imprimió el enfoque interaccionista al estudio.

Este artículo presenta la descripción de la categoría -hallada en el estudio- de hacer frente al síntoma o al signo.*

* Por razones de exposición y para facilitar la lectura del texto, se utilizará el termino sintoma -lo que una persona siente- con la suposición de que incluye también el signo -lo que se manifiesta de manera visible. 


\section{Resultados}

Uno de los primeros temas que surgieron durante el análisis fue el uso que las mujeres hacían de los servicios médicos. Aparecieron cuestiones aparentemente contradictorias en los datos: mientras unas mujeres afirmaban que iban al médico "cuando no podían más", otras, por el contrario, acudían al "primer síntoma", y otras más no consideraban siquiera ir al médico, pues no creían que ello pudiera ayudarlas.

Para comprender el fenómeno, los datos se interpretaron y organizaron alrededor de la categoría "Proceso de enfrentar el síntoma"; la teoría interaccionista ${ }^{17}$ y estudios previos guiaron este análisis. Se encontró que el proceso de enfrentar el síntoma comprende tres momentos: a) valoración del síntoma, b) medición de capacidades y evaluación de opciones, y c) selección de ayuda (figura 1).

\section{Valoración del síntoma: inicio del proceso}

El proceso de enfrentar el síntoma se inicia con su valoración; aquí se interpreta el significado del síntoma, es decir, se le atribuye importancia y se le clasifica. Los datos de las entrevistas han mostrado que se valora el síntoma según los parámetros de gravedad y de intensidad; así, se hablaba de que es un dolor "horrible", o, por el contrario, que no es "nada serio". También se valora la persistencia del síntoma, su evolución y normalidad. Las mujeres entrevistadas hacían referencia a padecimientos que "no se quitan", que van "a peor" o que son algo "normal" y "se quitan". Las mujeres utilizan la pérdida de funcionalidad ${ }^{18}$ como otro criterio para atribuir importancia a su síntoma. Por ejemplo, una mujer acudió al médico cuando ya "no podía hacer nada" con su mano derecha: había intentado todo tipo de autocuidados hasta que no pudo mover su mano, lo que le impedía trabajar. Por lo tanto, en el proceso de valoración del síntoma no se toma en cuenta sólo un parámetro, sino varios. Cuando iba al médico, una mujer comentó:

Algún dolor fuerte en un sitio de mi cuerpo, que no es común, digo: “¿Por qué me estará doliendo en esta área? ¿Qué será lo que tengo?, ya llevo tantos días y no se me ha quitado". Entonces de una vez voy al médico (Claudia).*

\footnotetext{
* Las cursivas son de la autora.
}

El objetivo de la valoración es, por tanto, determinar la necesidad de buscar ayuda. No obstante, esta valoración es subjetiva y se hace comparando las variaciones del propio estado de salud en un tiempo determinado, o bien, en relación con el de otras personas, por lo que la importancia que se le atribuye al síntoma depende de la percepción y del contexto de cada quien. ${ }^{3}$ Así, se ha señalado que lo que una persona reconoce como síntoma es, en parte, una desviación del modelo de normalidad ${ }^{19}$ establecido por la experiencia cotidiana, que varía cultural e históricamente. El sistema de creencias respecto a la salud es aquí un recurso; según la cultura, hay enfermedades que no se comunican al médico no porque no se perciban como tales, sino porque hay criterios diferentes acerca de su gravedad. ${ }^{4}$

La cita anterior apunta hacia otra actividad que está muy unida a este proceso de valoración: la explicación del síntoma o, como Mechanic ${ }^{20}$ identificó, "la atribución de causa". Esto tiene mucha importancia, pues dirige los autocuidados o el tipo de ayuda que se busca; algunas mujeres comentaron que van directamente a un especialista o que saben lo que deben tomar para aliviar el malestar. La explicación del síntoma también determina si se busca ayuda. Efectivamente, los datos muestran que, cuando se tiene una explicación para el síntoma, no se piensa que es necesario acudir al médico. Así, Marta, quien no recuperaba peso, comentó durante la entrevista que no fue al médico porque sabía que la razón era una mala alimentación. Otros estudios que han explorado las razones por las cuales los enfermos se retrasan en ir al médico han señalado también la importancia que tiene la etiología que percibe el enfermo. ${ }^{3}$

\section{Medición de capacidad y evaluación de opciones: etapa intermedia}

En el proceso de buscar ayuda hay una etapa intermedia entre la valoración del síntoma y la selección de ayuda, que es la medición de la capacidad y la evaluación de opciones. Los datos indican que las mujeres también valoran si tienen la capacidad para hacerse cargo de su dolencia y, además, anticipan el costo que esto puede significar. Aquí la capacidad se refiere tanto a cuestiones socioeconómicas (el nivel adquisitivo y el acceso a la ayuda) como a las fuerzas para autocuidarse.

Al sopesar las opciones de autocuidarse o de buscar ayuda, las mujeres calculan el costo que esto puede significar para su salud: 
[...] me preocupa cuando estoy enferma, y si me toca ir donde el médico, pues voy. No puedo pasar eso como inadvertido, puede ser un costo para mi salud o una intervención, una operación [...] (Alejandra).*

Esta cita indica que se valoran las consecuencias de no buscar ayuda, ya que se prevé el costo que ello puede llegar a suponer en caso de que se complique o agrave la condición. En este cálculo interviene la experiencia que se ha tenido con una dolencia. Alejandra explicó que tuvo que ser operada y por eso nunca más en su vida se volvería a descuidar. La bibliografía señala que al sopesar los costos y beneficios de buscar o no ayuda médica se involucran factores sociales, temas prácticos y preocupaciones económicas. ${ }^{1}$ Así, se puede decir que en esta etapa intermedia se contextualiza la posibilidad de buscar o no ayuda. El hecho de que el síntoma impida el desarrollo de las actividades, aunque importante -como se ha indicado- ${ }^{21}$ no es el único criterio.

\section{Selección del tipo de ayuda: etapa final}

El proceso finaliza con la seleccion del tipo de ayuda. Aquí se decide entre la ayuda profesional, la legal o el autocuidado; se pasa de valorar la necesidad de buscar ayuda a valorar cuál es la más apropiada.

Cuando se decide buscar la ayuda de un profesional de la salud, implícitamente se valora su competencia y capacidad para resolver el problema. Una mujer comentó que "uno tiene que saber a quién se busca" (Cecilia). En la valoración de la competencia médica pueden intervenir diversos factores. Freidson, ${ }^{19}$ por ejemplo, identifica factores de clase social y encuentra que la clase media en Estados Unidos de América va más al médico. No obstante, los datos de este estudio indican la importancia que tiene la confianza en el profesional de la salud, las relaciones que se establezcan con él y la propia experiencia de la enfermedad. Esto explica por qué en los datos de este estudio hay mujeres de clase media que no acuden al médico, y otras de clase trabajadora que sí lo hacen. Claramente, una mujer durante la entrevista comentó que va poco "donde los médicos" porque no cree en ellos.

Es importante resaltar que, aunque se haya decidido acudir al médico, las mujeres no siempre le comunican todos los síntomas o padecimientos. Se selecciona también el motivo de consulta:

\footnotetext{
* Las cursivas son de la autora.
}

Entonces yo fui donde el médico (a un chequeo) y le dije: "Doctor, yo no siento nada", claro que la enfermedad del colon ni siquiera se la comenté porque yo siento que es eso, o porque siempre me han dicho que es eso. Yo la manejo [...] (Alba).

Esta cita muestra que el autocuidado está presente en todo el proceso de búsqueda de ayuda. Se ha señalado que los remedios caseros se usan al mismo tiempo que la terapéutica facultativa. ${ }^{21}$ Una razón puede ser, como se comenta en la cita, que se consulte sólo por aquellas dolencias que no se pueden manejar.

Una vez que se acude a un profesional de la salud, se entra en un circuito que en la medicina facultativa puede significar consultas a otros especialistas o el ingreso a un hospital. A este proceso le acompaña un trabajo de salud, definido como el esfuerzo que las mujeres realizan para lograr, mantener o recuperar su bienestar. Cabe aclarar que se trata de un concepto más amplio que el de autocuidado terapéutico, pues se puede hablar de que hay un trabajo de salud cuando la persona toma adecuadamente lo que le ha sido medicado. ${ }^{22}$ Este circuito puede finalizar en el autocuidado, como es el caso de una enfermedad crónica.

Las mujeres también recurren a familiares y amigos para resolver sus problemas de salud. De esta manera, hacen uso de lo que se denomina en este estudio circuito lego, pues se hace un recorrido entre personas y tratamientos para resolver una dolencia o un síntoma. La confianza es también aquí un tema decisivo:

A veces me da más confianza el amigo que el profesional, pues es que el profesional a veces tiene una capa [...] (Lucero).

El circuito lego funciona de manera similar al profesional: se diagnostica, se trata y se deriva. Así, cuando el tratamiento o los remedios aconsejados no surten efecto, el sistema lego deriva al médico; se transita de los cuidados informales a los formales. Esto es consistente con lo descrito en la literatura como sistema lego de derivacion, donde es la cultura lega, y no los valores profesionales, la que define el significado de la enfermedad. ${ }^{19}$ Los datos de este estudio muestran además que la red social refuerza el seguimiento del tratamiento que prescribió el profesional. Así, se recuerda tomar el medicamento o se apoya el cumplimiento de una terapéutica difícil.

Por último, en este proceso de enfrentar el síntoma se puede optar por el autocuidado, ya sea por no tener confianza en los circuitos profesionales o en los legos o porque ambos no estén accesibles. En el caso 
de que el síntoma no desaparezca, el proceso se reinicia, como se indica en la figura 1.

\section{Discusión}

La literatura sobre el tema muestra que el proceso por el cual el enfermo busca ayuda es activo, que implica la toma de diversas decisiones y cubre diversas etapas. ${ }^{1,6,10,18,20}$ En el presente estudio se describió un proceso de valoración del síntoma y de selección del tipo de ayuda que permitió develar algunos de los parámetros y criterios que utilizan las mujeres para tomar la decisión de buscar ayuda o no. Además, se revela que las mujeres valoran sus propias capacidades y las posibles consecuencias de sus decisiones. Debido a las limitaciones del estudio, no se pueden generalizar sus resultados; sin embargo, se espera que permitan una mejor comprensión del camino que recorren las personas al sentirse enfermas.

Se ha documentado que en casi todas las culturas la mayor parte de la atención primaria tiene lugar dentro de la familia, en lo que se denomina sector popular de la atención, y que las mujeres son los principales proveedores. ${ }^{3}$ El estudio señala el papel que tiene la red informal de cuidados en la prestación de atención y en la derivación al sistema formal. Aunque se tienda a pensar que la escasa utilización de los servicios de salud significa que no se cubren necesidades, ${ }^{23}$ esto

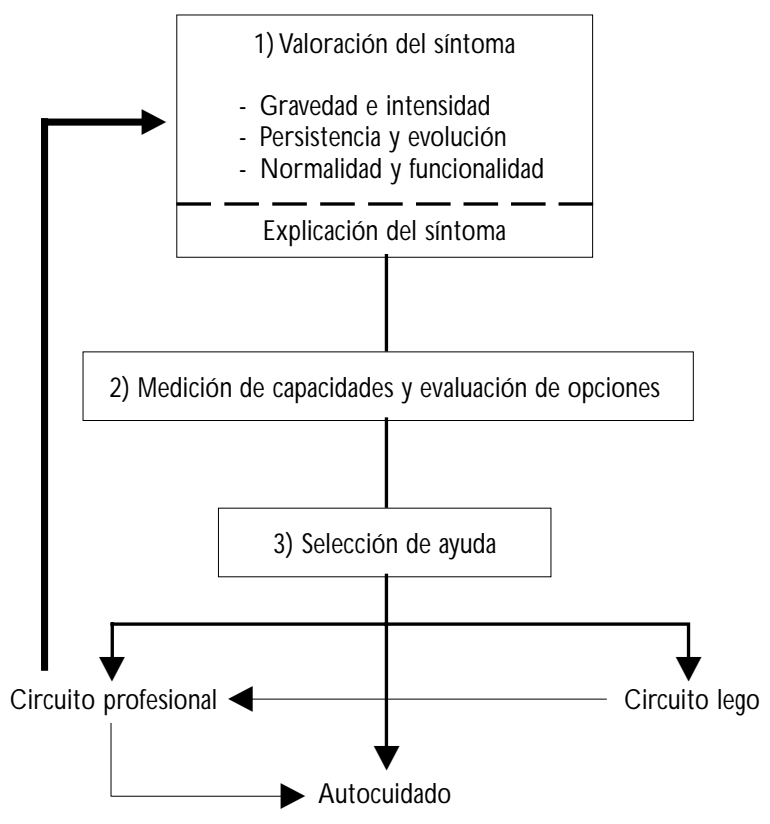

Figura 1. Proceso de enfrentar el síntoma no siempre es así: las necesidades de salud se pueden estar resolviendo en este sector popular.

Las investigaciones cualitativas permiten mejorar la comprensión de los problemas investigados y pueden proporcionar dirección a las acciones dirigidas a la salud. ${ }^{24}$ Este estudio muestra que las mujeres son activas en el diagnóstico y en la solución de sus problemas de salud, pues continuamente están tomando decisiones relativas al origen y a la naturaleza de sus dolencias y a la resolución de las mismas. El encuentro con los profesionales es el resultado de un proceso complejo en el que las mujeres han interpretado, decidido y seleccionado un tipo de ayuda para sus problemas de salud. En este sentido, es necesario que los profesionales de la salud tengan sensibilidad hacia los relatos subjetivos de los pacientes y comprendan de manera informada la diversidad de creencias y significados que atribuyen a su salud. Además, para que se den las tan recomendadas relaciones terapéuticas entre los pacientes y los profesionales, ${ }^{25}$ se hace imprescindible que se reconozca el conocimiento del paciente en cuanto a su padecimiento y que no se desestime su participación real en la recuperación de su salud. Específicamente, no se debe olvidar que las mujeres son las principales productoras de salud, ${ }^{26}$ por lo que es necesario trabajar conjuntamente con ellas para maximizar este potencial.

El papel crucial que cumple el circuito lego en la prestación de cuidados y en la derivación pone de manifiesto que las personas no son simplemente consumidoras de servicios de salud; concretamente, se ha sugerido que la red de parientes debe considerarse educadora en aspectos relativos a la salud. ${ }^{4}$ Los profesionales de la salud pública deben apoyar y orientar el papel del círculo familiar, de las amistades y de la comunidad en general en la atención primaria.

Por último, se espera que, con la descripción de la categoría "hacer frente al síntoma", los profesionales de la salud se interesen por comprender aún más cómo experimentan la enfermedad los pacientes, y contribuyan a contextualizar las acciones preventivas y de promoción de la salud.

\section{Referencias}

1. Jones LJ. The social context of health and health work. Houndsmills: MacMillan, 1994.

2. Fitzpatrick R. Lay concepts of illness. En: Fitzpatrick R, Hinton J, N ewman S, Scambler G, Thompson J, comp.The experience of illness. Londres: Tavistock, 1984:11-31.

3. Helman C. Culture, health, and illness. 2a. edición. Londres:W right, 1990. 
4. Freund PES, McG uire MB. Health, illness, and the social body. 2a. edición, Englewood C liffs: Prentice-Hall, 1995.

5. Coe RM. Sociología de la medicina. 2a. edición. Madrid:Alianza, 1979.

6.Taylor S, Field D. Sociology of health and health care. 0 xford: Blackwell, 1993.

7. Dirección Seccional de Salud. Mujeres y salud: un estudio cualitativo. Medellín: Dirección Seccional de Salud, 1995.

8. Tuckett D. Introduction. En:Tuckett D, comp. An introduction to medical sociology. Londres:Tavistock, 1976:3-40.

9. Scambler G, Scambler A.The illness iceberg and aspects of consulting behaviour. En: Fitzpatrick R, Hinton J, N ew man S, Scambler G, Thompson J, comp. The experience of illness. Londres:Tavistock, 1984:32-53.

10.Tuckett D. Becoming a patient. En:Tuckett D, comp. An introduction to medical sociology. Londres:Tavistock, 1976:169-189.

11. Lincoln IS. Sympathetic connections between qualitative methods and health research. Q ual Health Res 1992;2:375-391.

12. Strauss A, Corbin J. Basics of qualitative research-grounded theory procedures and techniques. N ewbury Park (CA): Sage, 1990.

13. Lincoln IA, Guba EG. N aturalistic inquiry. Beverly Hills (CA): SAGE, 1985.

14. Lofland J, Lofland HL.A nalyzing social settings. Belmont (CA):W adsworth, 1984.

15. Denzin N K. The research act. 2a. edición. N ueva York: MacG raw Hill, 1978.
16. G laser BA, Strauss AL. The discovery of grounded theory: Strategies for qualitative research. Chicago:Aldine Press, 1967.

17. Blumer H. Symbolic interactionism: Perspective and method. Englewood Cliffs: Prentice-Hall, 1969.

18. Zola IK. Pathways to the doctor-from person to patient. Soc Sci Med 1973;7:677-689

19. Freidson E. La profesión médica. Barcelona: Península, 1978.

20. Mechanic D.The concept of illness behaviour: Culture, situation, and personal disposition. Psychol Med 1986;16:1-7.

21. Schapira M. Representación del proceso de salud-enfermedad y la valoración de la atención médica desde la perspectiva de la mujer. Cuadernos Médico Sociales (Rosario) 1993:41-66.

22. De la Cuesta C. Trabajo de salud. Enfermería Clínica 1996:6:95-101.

23. 0 choa-Díaz López H, Sánchez-Pérez HJ, Martínez-Guzmán LA. U so de un índice de bienestar social para la planificación de la salud a nivel municipal. Salud Publica Mex 1996;38:257-267.

24. Hammersley M.W hat's wrong with ethnography? Londres: Routledge, 1992.

25. C ampero-C uenca L. Calidad de la relación pedagógica médico-paciente en consulta externa: el caso de 0 axaca, México. Salud Publica Mex 1990;32:192-204.

26. Stacey M. The power of lay knowledge: A personal view. En: Popay J, W illiams $G$, comp. Researching the people's health. Londres: Routledge, 1994:85-98. 